Proceedings of the 2009 Winter Simulation Conference

M. D. Rossetti, R. R. Hill, B. Johansson, A. Dunkin, and R. G. Ingalls, eds.

\title{
MULTILEVEL MONTE CARLO FOR BASKET OPTIONS
}

\author{
Michael B. Giles \\ Oxford-Man Institute of Quantitative Finance \\ Oxford University Mathematical Institute \\ Oxford \\ United Kingdom
}

\begin{abstract}
The multilevel Monte Carlo method has been previously introduced for the efficient pricing of options based on a single underlying quantity. In this paper we show that the method is easily extended to basket options based on a weighted average of several underlying quantities. Numerical results for Asian, lookback, barrier and digital basket options demonstrate that the computational cost to achieve a root-mean-square error of $\varepsilon$ is $O\left(\varepsilon^{-2}\right)$. This is achieved through a careful construction of the multilevel estimator which computes the difference in expected payoff when using different numbers of timesteps.
\end{abstract}

\section{INTRODUCTION}

Giles (Giles 2007, Giles 2008) has recently introduced a multilevel Monte Carlo path simulation method which improves the efficiency of financial option pricing by combining results using different numbers of timesteps. This can be viewed as a generalisation of the two-level method of Kebaier (Kebaier 2005) and is also similar in approach to Heinrich's multilevel method for parametric integration (Heinrich 2001). Reference (Giles 2008) introduced the multilevel Monte Carlo method and proved that it can lower the computational complexity of path-dependent Monte Carlo evaluations, while reference (Giles 2007) demonstrated that the computational cost can be further reduced by using the Milstein discretisation. In this paper we briefly review the key ideas and show that the same approach can be used for basket options in which the financial payoff functions depends on a weighted averaged of a number of underlying assets.

\section{MULTILEVEL MONTE CARLO METHOD}

We start by considering a scalar SDE with general drift and volatility terms,

$$
\mathrm{d} S(t)=a(S, t) \mathrm{d} t+b(S, t) \mathrm{d} W(t), \quad 0<t<T
$$

with given initial data $S_{0}$. In the case of European and digital options, we are interested in the expected value of a function of the terminal state, $f(S(T))$, but in other cases the valuation depends on the entire path $S(t), 0<t<T$. Using a simple Euler-Maruyama discretisation with first order weak convergence, to achieve a r.m.s. error of $\varepsilon$ would require $O\left(\varepsilon^{-2}\right)$ independent paths, each with $O\left(\varepsilon^{-1}\right)$ timesteps, giving a computational complexity which is $O\left(\varepsilon^{-3}\right)$.

Consider performing Monte Carlo path simulations with different numbers of uniform timesteps $h_{l}=2^{-l} T, l=0,1, \ldots, L$, so on the coarsest level, $l=0$, the simulations use just 1 timestep, while on the finest level, $l=L$, the simulations use $2^{L}$ timesteps. For a given Brownian path $W(t)$, let $P$ denote the payoff, and let $\widehat{P}_{l}$ denote its approximation using a numerical discretisation with timestep $h_{l}$. Because of the linearity of the expectation operator, it is clearly true that

$$
\mathbb{E}\left[\widehat{P}_{L}\right]=\mathbb{E}\left[\widehat{P}_{0}\right]+\sum_{l=1}^{L} \mathbb{E}\left[\widehat{P}_{l}-\widehat{P}_{l-1}\right]
$$


This expresses the expectation on the finest level as being equal to the expectation on the coarsest level plus a sum of corrections which give the difference in expectation between simulations using different numbers of timesteps. In the multilevel method we independently estimate each of the expectations on the right-hand side in a way which minimises the overall variance for a given computational cost.

The simplest estimator for $\mathbb{E}\left[\widehat{P}_{l}-\widehat{P}_{l-1}\right]$ for $l>0$ is a mean of $N_{l}$ independent samples,

$$
\widehat{Y}_{l}=N_{l}^{-1} \sum_{i=1}^{N_{l}}\left(\widehat{P}_{l}^{(i)}-\widehat{P}_{l-1}^{(i)}\right)
$$

The key point here is that the quantity $\widehat{P}_{l}^{(i)}-\widehat{P}_{l-1}^{(i)}$ comes from two discrete approximations with different timesteps but the same Brownian path. The variance of this simple estimator is $\mathbb{V}\left[\widehat{Y}_{l}\right]=N_{l}^{-1} V_{l}$ where $V_{l}$ is the variance of a single sample. Combining this with independent estimators for each of the other levels, and with $\widehat{Y}_{0}$ being the usual estimate for $\mathbb{E}\left[\widehat{P}_{0}\right]$, the variance of the combined estimator $\widehat{Y}=\sum_{l=0}^{L} \widehat{Y}_{l}$ is $\mathbb{V}[\widehat{Y}]=\sum_{l=0}^{L} N_{l}^{-1} V_{l}$, while its computational cost is proportional to $\sum_{l=0}^{L} N_{l} h_{l}^{-1}$. Treating the $N_{l}$ as continuous variables, the variance is minimised for a fixed computational cost by choosing $N_{l}$ to be proportional to $\sqrt{V_{l} h_{l}}$.

The Euler-Maruyama discretisation gives $O\left(h^{1 / 2}\right)$ strong convergence, provided $a(S, t)$ and $b(S, t)$ satisfy certain conditions (Kloeden and Platen 1992). From this it follows that $\mathbb{V}\left[\widehat{P}_{l}-P\right]=O\left(h_{l}\right)$ for a European option with a Lipschitz continuous payoff. Hence for the simple estimator (3), the single sample variance $V_{l}$ is $O\left(h_{l}\right)$, and the optimal choice for $N_{l}$ is asymptotically proportional to $h_{l}$. Setting $N_{l}=O\left(\varepsilon^{-2} L h_{l}\right)$, the variance of the combined estimator $\widehat{Y}$ is $O\left(\varepsilon^{2}\right)$. If $L$ is chosen such that $L=\log \varepsilon^{-1} / \log 2+O(1)$, as $\varepsilon \rightarrow 0$, then $h_{L}=2^{-L}=O(\varepsilon)$, and so the bias error $\mathbb{E}\left[\widehat{P}_{L}-P\right]$ is $O(\varepsilon)$ due to standard results on weak convergence. Consequently, we obtain a Mean Square Error which is $O\left(\varepsilon^{2}\right)$, with a computational complexity which is $O\left(\varepsilon^{-2} L^{2}\right)=O\left(\varepsilon^{-2}(\log \varepsilon)^{2}\right)$.

This analysis is generalised in the following theorem (Giles 2008):

Theorem 1. Let $P$ denote a functional of the solution of stochastic differential equation (1) for a given Brownian path $W(t)$, and let $\widehat{P}_{l}$ denote the corresponding approximation using a numerical discretisation with timestep $h_{l}=M^{-l} T$.

If there exist independent estimators $\widehat{Y}_{l}$ based on $N_{l}$ Monte Carlo samples, and positive constants $\alpha \geq \frac{1}{2}, \beta, c_{1}, c_{2}, c_{3}$ such that

$\begin{aligned} \text { i) } & \left|\mathbb{E}\left[\widehat{P}_{l}-P\right]\right| \leq c_{1} h_{l}^{\alpha} \\ \text { ii) } & \mathbb{E}\left[\widehat{Y}_{l}\right]= \begin{cases}\mathbb{E}\left[\widehat{P}_{0}\right], & l=0 \\ \mathbb{E}\left[\widehat{P}_{l}-\widehat{P}_{l-1}\right], & l>0\end{cases} \\ \text { iii) } & \mathbb{V}\left[\widehat{Y}_{l}\right] \leq c_{2} N_{l}^{-1} h_{l}^{\beta}\end{aligned}$

iv) $C_{l}$, the computational complexity of $\widehat{Y}_{l}$, is bounded by $C_{l} \leq c_{3} N_{l} h_{l}^{-1}$,

then there exists a positive constant $c_{4}$ such that for any $\varepsilon<e^{-1}$ there are values $L$ and $N_{l}$ for which the multilevel estimator

$$
\widehat{Y}=\sum_{l=0}^{L} \widehat{Y}_{l}
$$

has a mean-square-error with bound

$$
\mathbb{E}\left[(\widehat{Y}-\mathbb{E}[P])^{2}\right]<\varepsilon^{2}
$$

with a computational complexity $C$ with bound

$$
C \leq \begin{cases}c_{4} \varepsilon^{-2}, & \beta>1, \\ c_{4} \varepsilon^{-2}(\log \varepsilon)^{2}, & \beta=1, \\ c_{4} \varepsilon^{-2-(1-\beta) / \alpha}, & 0<\beta<1 .\end{cases}
$$




\section{Giles}

\section{MILSTEIN DISCRETISATION}

The Milstein discretisation of equation (1) is

$$
\widehat{S}_{n+1}=\widehat{S}_{n}+a_{n} h+b_{n} \Delta W_{n}+\frac{1}{2} \frac{\partial b_{n}}{\partial S} b_{n}\left(\left(\Delta W_{n}\right)^{2}-h\right)
$$

In the above equation, the subscript $n$ is used to denote the timestep index, and $a_{n}, b_{n}$ and $\partial b_{n} / \partial S$ are evaluated at $\widehat{S}_{n}, t_{n}$. Provided certain conditions are satisfied (Kloeden and Platen 1992), the Milstein scheme gives $O(h)$ strong convergence, and for a Lipschitz European payoff this immediately leads to the result that $V_{l}=O\left(h_{l}^{2}\right)$.

Reference (Giles 2007) addresses the tougher challenges of Asian, lookback, barrier and digital options. The key to the Asian, lookback and barrier option constructions is a conditional piecewise Brownian interpolation. Within the time interval $\left[t_{n}, t_{n+1}\right]$ we approximate the drift and volatility as being constant with the interval and use a Brownian interpolation conditional on the two end values $\widehat{S}_{n}$ and $\widehat{S}_{n+1}$, giving

$$
\widehat{S}(t)=\widehat{S}_{n}+\lambda(t)\left(\widehat{S}_{n+1}-\widehat{S}_{n}\right)+b_{n}\left(W(t)-W_{n}-\lambda(t)\left(W_{n+1}-W_{n}\right)\right)
$$

where $\lambda(t)=\left(t-t_{n}\right) /\left(t_{n+1}-t_{n}\right)$. Standard results for the distribution of the extrema and averages of Brownian motions (Glasserman 2004) can then be used to construct suitable multilevel estimators (Giles 2007).

Similarly, for the digital option which has a discontinuous payoff, one can use a constant coefficient Brownian extrapolation conditional on the value $\widehat{S}_{N-1}$, one timestep before the end. Following an approach used for payoff smoothing for pathwise sensitivity analysis (Glasserman 2004), the conditional expectation for the payoff can be evaluated analytically and this is then used to construct the multilevel estimator (Giles 2007).

\section{BASKET OPTIONS}

In basket options the value is dependent on a weighted average of $J$ underlying assets, $\bar{S}(t)=\sum_{j=1}^{J} \mu_{j} S_{j}(t)$, each of which satisfies an SDE of the form (1) driven by Brownian motions $W_{j}(t)$ with correlation matrix $\Sigma$. In constructing the multilevel estimator, the important observation is that the average of the Brownian interpolations for the $J$ underlying assets gives

$$
\begin{aligned}
\overline{\widehat{S}}(t) & =\overline{\widehat{S}}_{n}+\lambda(t)\left(\overline{\widehat{S}}_{n+1}-\overline{\widehat{S}}_{n}\right)+\sum_{j=1}^{J} \mu_{j} b_{j, n}\left(W_{j}(t)-W_{j, n}-\lambda(t)\left(W_{j, n+1}-W_{j, n}\right)\right) \\
& =\overline{\widehat{S}}_{n}+\lambda(t)\left(\overline{\widehat{S}}_{n+1}-\overline{\widehat{S}}_{n}\right)+\bar{b}_{n}\left(W(t)-W_{n}-\lambda(t)\left(W_{n+1}-W_{n}\right)\right),
\end{aligned}
$$

where $W(t)$ is another Brownian motion which is a weighted average of the $W_{j}(t)$, and $\bar{b}_{n}$ is defined by

$$
\bar{b}_{n}^{2}=\sum_{i, j} \mu_{i} b_{i, n} \Sigma_{i, j} \mu_{j} b_{j, n}
$$

Since the Brownian interpolation for the basket average has the same form as the scalar interpolation, the multilevel estimators can be constructed in exactly the same way as in (Giles 2007) using $\bar{b}_{n}$.

The numerical results to be presented are for a basket of five assets, each modelled as a geometric Brownian motion:

$$
\mathrm{d} S_{j}=r S_{j} \mathrm{~d} t+\sigma_{j} S_{j} \mathrm{~d} W_{j}(t), \quad 0<t<T
$$

using a constant risk-free interest rate $r=0.05$, and five volatilities $\sigma=0.2,0.25,0.3,0.35,0.4$. The initial asset values are $S_{j}(0)=100$, the simulation interval is taken to be $T=1$, and the driving Brownian motions have a correlation of 0.25 . In each case, the option price is based on a simple arithmetic average of the five assets. 

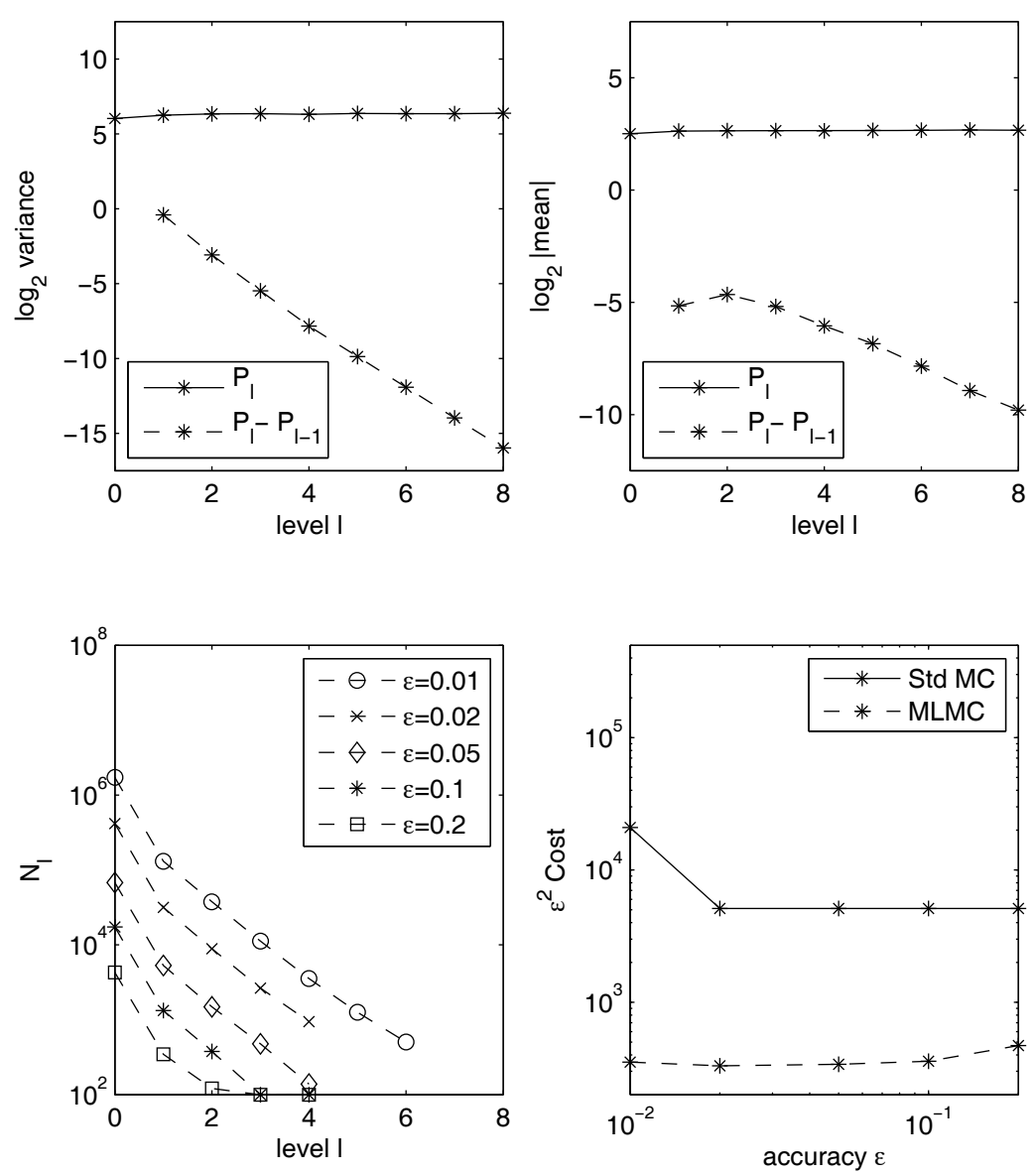

Figure 1: Asian option

\subsection{Asian Option}

The Asian basket option has the discounted payoff $P=\exp (-r T) \max (0, \overline{\bar{S}}-K)$, where $\overline{\bar{S}}$ is the time-average of the average of the underlying assets, and the strike is $K=100$.

The top left plot in Figure 1 shows the behaviour of the variance of both $\widehat{P}_{l}$ and $\widehat{P}_{l}-\widehat{P}_{l-1}$. The slope of the latter is approaching a value approximately equal to -2 , indicating that $V_{l}=O\left(h_{l}^{2}\right)$. On level $l=2$, which has just 4 timesteps, $V_{l}$ is already almost 1000 times smaller than the variance $\mathbb{V}\left[\widehat{P}_{l}\right]$ of the standard Monte Carlo method with the same timestep. The top right plot shows that $\mathbb{E}\left[\widehat{P}_{l}-\widehat{P}_{l-1}\right]$ is approximately $O\left(h_{l}\right)$, corresponding to first order weak convergence. This is used to determine the number of levels that are required to reduce the bias to an acceptable level (Giles 2008).

The bottom two plots have results from five multilevel calculations for different values of $\varepsilon$. Each line in the bottom left plot shows the values for $N_{l}, l=0, \ldots, L$, with the values decreasing with $l$ because of the decrease in both $V_{l}$ and $h_{l}$. It can also be seen that the value for $L$, the maximum level of timestep refinement, increases as the value for $\varepsilon$ decreases, requiring a lower bias error (Giles 2008). The bottom right plot shows the variation with $\varepsilon$ of $\varepsilon^{2} C$ where the computational complexity $C$ is defined as $C=\sum_{l} 2^{l} N_{l}$, which is the total number of fine grid timesteps on all levels. One line shows the results for the multilevel calculation and the other shows the corresponding cost of a standard Monte Carlo simulation of the same accuracy, i.e. the same bias error corresponding to the same value for $L$, and the same variance. It can be seen that $\varepsilon^{2} C$ is almost constant for the multilevel method, as expected, whereas for the standard Monte Carlo method it increases with $L$. For the most accurate case, $\varepsilon=0.01$, the multilevel method is approximately 100 times more efficient than the standard method. 

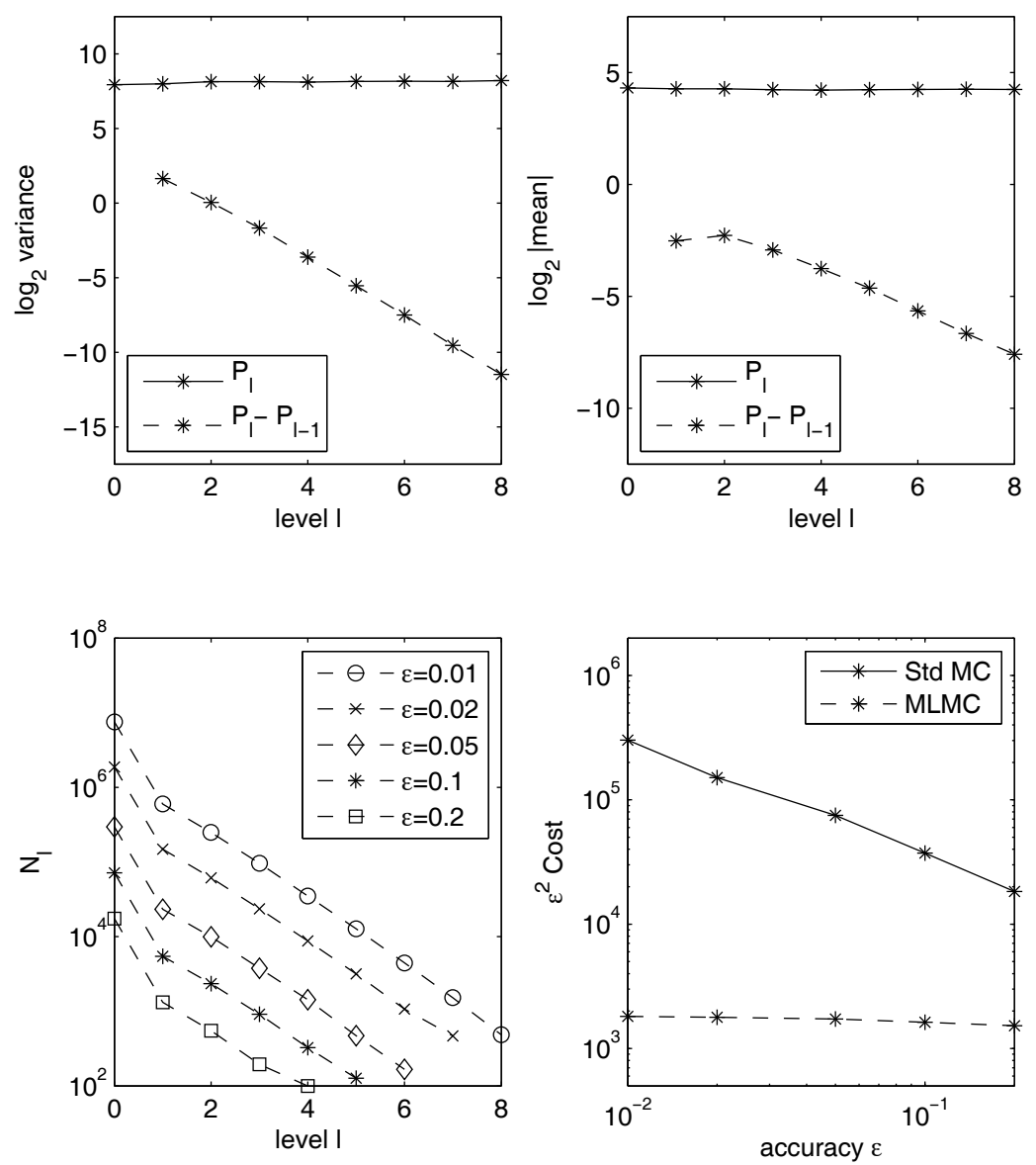

Figure 2: Lookback option

\subsection{Lookback Option}

The basket lookback option we consider has the discounted payoff $P=\exp (-r T)\left(\bar{S}(T)-\min _{0<t<T} \bar{S}(t)\right)$.

The top left plot in Figure 2 shows that the variance is $O\left(h_{l}^{2}\right)$, while the top right plot shows that the mean correction is $O\left(h_{l}\right)$. The bottom left plot shows that more levels are required to reduce the discretisation bias to the required level. Consequently, the savings relative to the standard Monte Carlo treatment are greater, up to a factor of approximately 150 for $\varepsilon=0.01$. The computational cost of the multilevel method is almost perfectly proportional to $\varepsilon^{-2}$.

\subsection{Barrier Option}

The barrier option which is considered is a down-and-out call with payoff $P=\exp (-r T)(\bar{S}(T)-K)^{+} \mathbf{1}_{\tau>T}$, where the notation $(\bar{S}(T)-K)^{+}$denotes $\max (0, \bar{S}(T)-K), \mathbf{1}_{\tau>T}$ is an indicator function taking value 1 if the argument is true, and zero otherwise, and the crossing time $\tau$ is defined as $\tau=\inf _{t>0}\{\bar{S}(t)<B\}$. The barrier value is taken to be $B=85$, and the strike is again $K=100$.

The top left plot in Figure 3 shows that the variance is approximately $O\left(h_{l}^{3 / 2}\right)$. The reason for this is that an $O\left(h_{l}^{1 / 2}\right)$ fraction of the paths have a minimum which lies within $O\left(h_{l}^{1 / 2}\right)$ of the barrier. In (Giles 2007) it is argued that for these paths the difference between the coarse and fine path payoff values is $O\left(h_{l}^{1 / 2}\right)$, giving a contribution to the overall variance which is $O\left(h_{l}^{3 / 2}\right)$. 

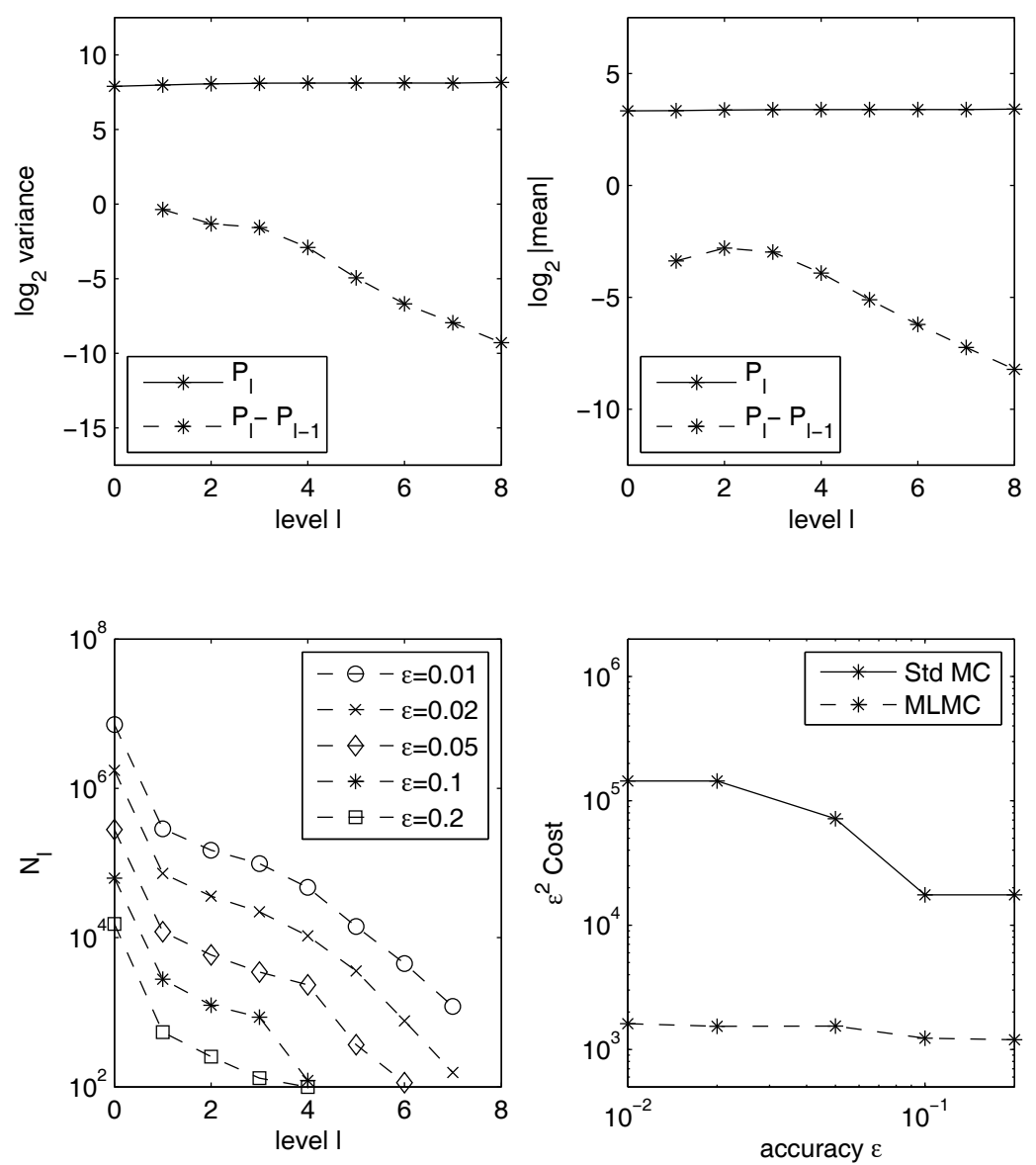

Figure 3: Barrier option

The top right plot shows that the mean correction is $O\left(h_{l}\right)$, corresponding to first order weak convergence. The bottom right plot shows that the computational cost of the multilevel method is again almost perfectly proportional to $\varepsilon^{-2}$, and for $\varepsilon=0.01$ it is 100 times more efficient that the standard Monte Carlo method.

\subsection{Digital Option}

The digital option has the discounted payoff $P=\exp (-r T) K \mathbf{1}_{S(T)>K}$ with strike $K=100$.

The top left plot in Figure 4 shows that the variance is approximately $O\left(h_{l}^{3 / 2}\right)$. The reason for this is similar to the argument for the barrier option. $O\left(h_{l}^{1 / 2}\right)$ of the paths have a minimum which lies within $O\left(h_{l}^{1 / 2}\right)$ of the strike. The fine path and coarse path trajectories differ by $O\left(h_{l}\right)$, due to the first order strong convergence of the Milstein scheme and this results in an $O\left(h_{l}^{1 / 2}\right)$ difference between the coarse and fine path evaluations.

One strikingly different feature is that the variance of the level 0 estimator is zero. This is because the multilevel treatment introduced in (Giles 2007) uses a conditional expectation (based on a simple Brownian extrapolation for which the expectation is known analytically) evaluated one timestep before the end. At level $l=0$ where there would usually be one timestep, there is no path simulation at all; one simply uses the analytic expression for the conditional expectation. This reduces the cost of the multilevel calculations even more than usual, giving more than a factor of 500 computational savings for $\varepsilon=0.01$. 

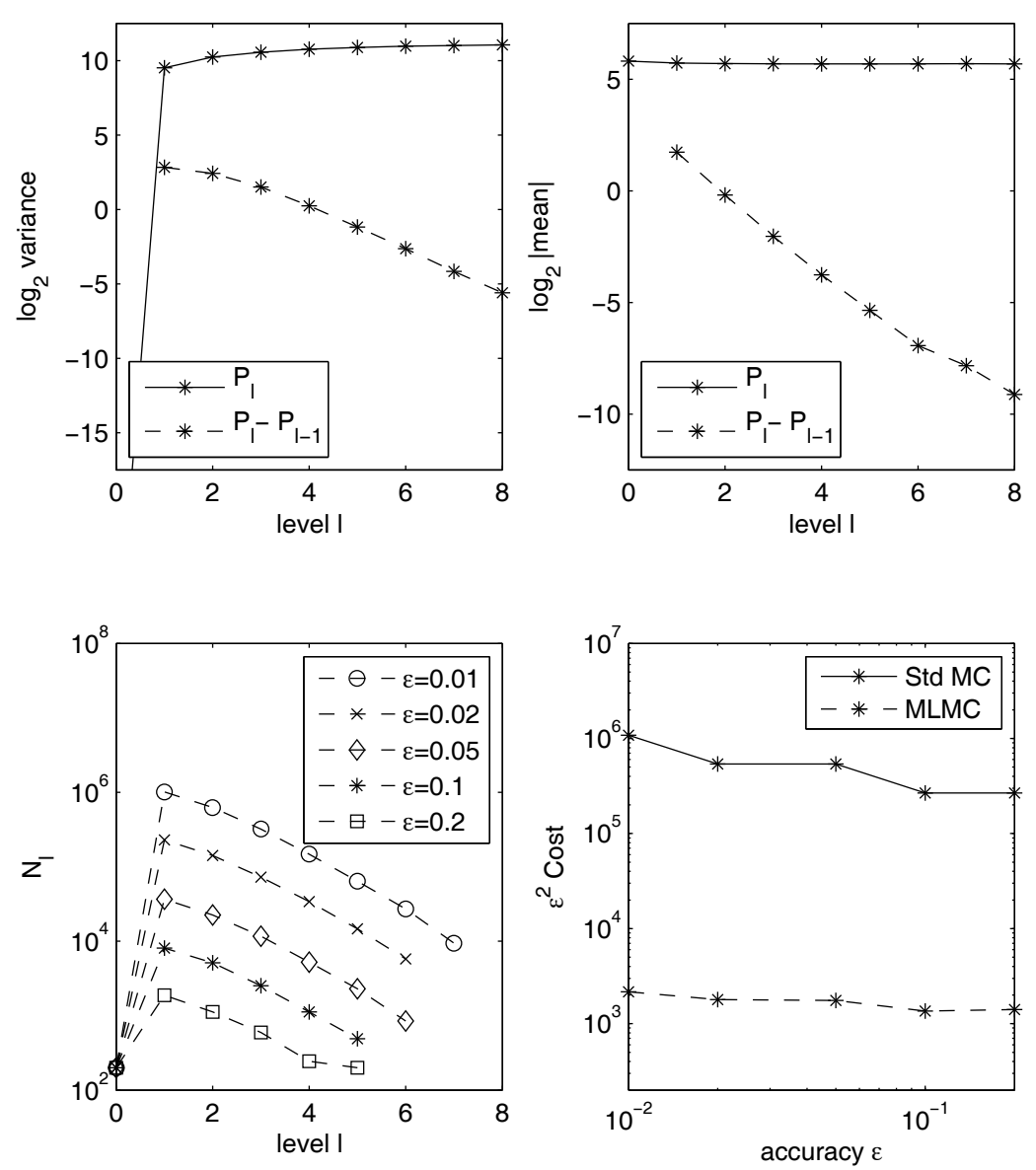

Figure 4: Digital option

\section{CONCLUSIONS}

In this paper we have reviewed the multilevel Monte Carlo method and have demonstrated that it achieves an $O\left(\varepsilon^{-2}\right)$ complexity when computing the value of basket options to within a root-mean-square error of $\varepsilon$. This built on the single asset methods introduced in (Giles 2007), by noting that the weighted average of a set of simple correlated Brownian motions is itself a simple Brownian motion, and so the same techniques can be applied as in the single asset case.

This paper does not present any numerical analysis of the techniques used. Previous work (Giles, Higham, and Mao 2009, Avikainen 2009) has analysed the multilevel method using the Euler-Maruyama discretisation. Current work by Giles, Debrabant and Rößler extending this analysis to the Milstein discretisation supports the orders of convergence demonstrated in this paper.

Future work will address the use of the multilevel approach for more general multivariate cases, in particular when the payoff function is a general discontinuous function of the underlying asset values at a set of discrete times. Other extensions to be considered are the computation of sensitivities (the "Greeks" in computational finance), and the application to general Lévy processes.

\section{ACKNOWLEDGMENTS}

This research has been supported by the Oxford-Man Institute of Quantitative Finance. 


\section{Giles}

\section{REFERENCES}

Avikainen, R. 2009. Convergence rates for approximations of functionals of SDEs. Finance and Stochastics 13 (3): $381-401$. Giles, M. 2007. Improved multilevel Monte Carlo convergence using the Milstein scheme. In Monte Carlo and Quasi-Monte Carlo Methods 2006, ed. A. Keller, S. Heinrich, and H. Niederreiter, 343-358. Springer-Verlag.

Giles, M. 2008. Multilevel Monte Carlo path simulation. Operations Research 56 (3): 981-986.

Giles, M., D. Higham, and X. Mao. 2009. Analysing multilevel Monte Carlo for options with non-globally Lipschitz payoff. Finance and Stochastics 13 (3): 403-413.

Glasserman, P. 2004. Monte Carlo methods in financial engineering. Springer, New York.

Heinrich, S. 2001. Multilevel Monte Carlo methods, Volume 2179 of Lecture Notes in Computer Science, 58-67. SpringerVerlag.

Kebaier, A. 2005. Statistical Romberg extrapolation: a new variance reduction method and applications to options pricing. Annals of Applied Probability 14 (4): 2681-2705.

Kloeden, P., and E. Platen. 1992. Numerical solution of stochastic differential equations. Springer, Berlin.

\section{AUTHOR BIOGRAPHY}

MICHAEL B. GILES is a Professor of Scientific Computing in the Oxford University Mathematical Institute, and a member of the Oxford-Man Institute of Quantitative Finance. He received his Ph.D. in Aeronautics and Astronautics from MIT and worked on computational fluid dynamics for many years at MIT and in the Oxford University Computing Laboratory before moving into computational finance. He serves as an Associate Editor for the Journal of Computational Finance and the SIAM Journal of Financial Mathematics. His email address is <mike.giles@maths.ox.ac.uk>. 